\title{
Neue Dimension in der klinischen Schmerztherapie
}

— Wenn Kinder einen kleinen Eingriff benötigen, für den eine Narkose viel zu aufwändig wäre, stellt sich die Frage, wie man stattdessen Schmerzen und Ängste erfolgreich verhindern kann. Hier bietet der Einsatz von LIVOPAN ${ }^{\circledR}$ eine neue, attraktive Lösung: Das Gasgemisch aus je 50\% Distickstoffmonoxid und Sauerstoff bewirkt eine schnelle und effektive, nicht invasive Schmerzlinderung bei kurzen Eingriffen mittlerer Schmerzintensität.

Erste Daten zum Einsatz von LIVOPAN ${ }^{\circledR}$ in der pädiatrischen Onkologie präsentierte Dr. Till Dresbach vom Zentrum für Kinderheilkunde der Universitätsklinik Bonn in Form eines Postervortrags auf der 105. Jahrestagung der Deutschen Gesellschaft für Kinderund Jugendmedizin Anfang September in Mannheim. Im Rahmen einer prospektiven Anwendungsbeobachtung hatten er und sein Team bei 37 onkologischen Patienten schmerzhafte Prozeduren, zumeist Lumbalpunktionen, unter inhalativer Analgesie mit LIVOPAN $^{\circledR}$ durchgeführt. Während des Eingriffs wurden die Vitalparameter gemessen und Nebenwirkungen dokumentiert. Im Anschluss daran wurden Patienten und Eltern zur Schmerzintensität und zur Zufriedenheit mit der Methode befragt.

Fast drei Viertel der Befragten (72\%) berichteten über keine oder geringe Schmerzen während des Eingriffs. Die einzigen Nebenwirkungen waren Müdigkeit (11\%) und Erbrechen (5\%). Entsprechend dieser Zahlen äußerten sich 88\% der Patienten „zufrieden“ oder sogar „sehr zufrieden“ mit der inhalativen Analgesie. Das Fazit von Dr. Dresbach: „Die inhalative Analgesie mit LIVOPAN ${ }^{\circledR}$ ist, wenn die Patienten die Maskeninhalation tolerieren, bei kurzzeitigen Eingriffen mittlerer Schmerzintensität eine sichere und effiziente Methode der Analgosedierung. Die atraumatische Applikation, der Wirkungseintritt innerhalb von nur 3-5 Minuten und die geringe Nebenwirkungsrate bedingen eine hohe Akzeptanz der Methode bei Patienten, Eltern und Personal.“

Nach Informationen von Linde Gas Therapeutics, Unterschleißheim

\section{Bewährtes Mukolytikum bei Bronchitis}

— Erkältungskrankheiten gehören zu den häufigsten Infektionen im Kindesalter und sind nicht selten mit einer Bronchitis verbunden. In den Atemwegen kommt es dabei zur verstärkten Produktion von mukösem Sekret, das nur schwer abgehustet werden kann. Zusätzlich begünstigt der festsitzende Schleim die Entstehung von bakteriellen Superinfektionen. Die kleinen Patienten leiden nicht nur an mitunter sehr schmerzhaftem Husten, sondern auch unter einem stark verminderten Allgemeinbefinden.

Ein zuverlässig wirkendes Mukolytikum kann hier rasch Erleichterung verschaffen. Es hilft, den zähen Schleim zu verflüssigen und leichter abzuhusten. Dabei hat sich Fluimucil ${ }^{\circledR}$ Kindersaft mit dem Wirkstoff Acetylcystein seit vielen Jahren bewährt. Der Kindersaft enthält ein Derivat der körpereigenen Aminosäure Cystein und wirkt auf zweifache Weise: Zum einen spaltet Acetylcystein die Disulfidbrücken im Schleim, der damit flüssiger wird und leichter abgehustet werden kann. Zum anderen unterstützt Acetylcystein als Precursormolekül von Glutathion die antioxidative Abwehr. So werden die im Rahmen der Entzündungsreaktion entstehenden freien Radikale rasch unschädlich gemacht. Die positive Wirkung des Präparats bei kindlicher Bronchitis ist in zahlreichen Studien untersucht worden. Fluimucil ${ }^{\circledR}$ Kindersaft mit seiner flüssigen Darreichungsform und dem Himbeergeschmack wird von Kindern gerne eingenommen. Je nach Alter des Kindes stehen drei kostengünstige Packungsgrößen zur Verfügung. Für Kinder unter zwölf Jahren ist dieser Kindersaft auch gemäß der neuen Arzneimittelrichtlinie erstattungsfähig. Für ältere Kinder und Erwachsene stehen Fluimucil ${ }^{\circledR}$ Brausetabletten mit Zitronen- oder Johannisbeergeschmack zur Verfügung.

Nach Informationen von Pierre Fabre Pharma, Freiburg

\section{Moderne Phytotherapie}

Phytotherapeutika, deren klinische Wirksamkeit wissenschaftlich nachgewiesen ist, sind eher die Ausnahme als die Regel. Nur durch Unternehmen wie Bionorica und ihren hohen Qualitätsanspruch gemäß dem Phytoneering-Prinzip werden moderne Herstellungs- und Analyse-

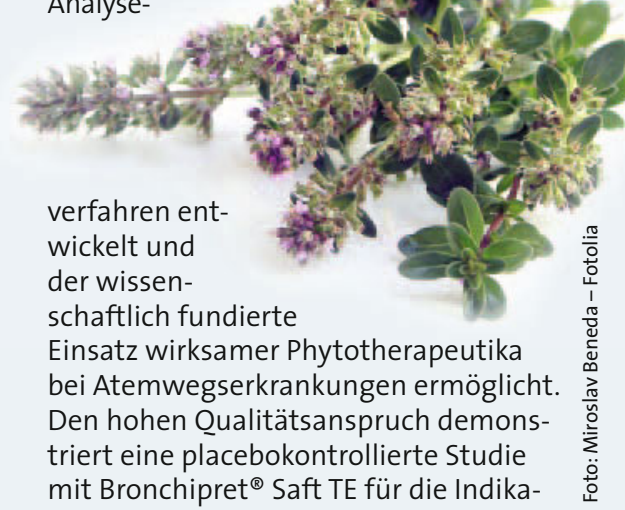
tion akute Bronchitis [Kemmerich B al. Arzneimittelforschung 2006; 56 : 652-60]. Sie kam zu dem Ergebnis, dass typische Beschwerden wie Hustenanfälle durch das Medikament zwei Tage früher zu 50\% reduziert wurden als unter Placebo. Es traten keinerlei schwerwiegende Nebenwirkungen auf.

Nach Informationen von Bionorica, Neumarkt

\section{Fixkombination nach wie vor erstattungsfähig}

Bei der Therapie mit Spasmo-Mucosolvan ${ }^{\circledR}$ - einer Fixkombination aus dem ß2-Sympathomimetikum Clenbuterol und dem Mukolytikum Ambroxol - steht die Behandlung spastischer Atemwegsverengungen im Vordergrund. Deshalb übernehmen die gesetzlichen Krankenkassen auch weiterhin die Kosten für ihre Versicherten. In der Arzneimittelrichtlinie des Gemeinsamen Bundesausschusses, die seit dem 1. April 2009 gilt, heißt es zwar, dass Hustenmittel von der Verordnungsfähigkeit ausgenommen sind, allerdings ist Spasmo-Mucosolvan ${ }^{\circledR}$ den in der Fachinformation angegebenen Anwendungsgebieten entsprechend zur Behandlung spastischer Verengungen der Atemwege auch weiterhin erstattungsfähig.

Nach Informationen von Boehringer Ingelheim, Ingelheim 